\title{
Theta-transforms and even zeta functions of Siegel modular forms
}

by

\author{
Anatoli Andrianov (St. Petersburg)
}

Introduction. We consider (holomorphic) Siegel modular forms on the upper half-plane

$$
\mathbb{H}=\mathbb{H}_{n}=\left\{Z=X+\left.i Y \in \mathbb{C}_{n}^{n}\right|^{\mathrm{t}} Z=Z, Y>0\right\}
$$

of genus $n$ such that

$$
F\left((A Z+B)(C Z+D)^{-1}\right)=\operatorname{det}(C Z+D)^{k} F(Z)
$$

for every matrix $\left(\begin{array}{ll}A & B \\ C & D\end{array}\right)$ of the group

$$
K=\Gamma_{0}^{n}(q)=\left\{\left(\begin{array}{cc}
A & B \\
C & D
\end{array}\right) \in \operatorname{Sp}_{n}(\mathbb{Z}) \mid C \equiv 0(\bmod q)\right\},
$$

where $n, k$, and $q$ are positive integers. All such functions form a finitedimensional linear space $\mathfrak{M}=\mathfrak{M}_{k}^{n}(q)$ over the field $\mathbb{C}$ of complex numbers. Each function of the space has Fourier expansion of the form

$$
F(Z)=\sum_{A \in \mathbb{E}, A \geq 0} f(A) \mathbf{e}(A Z) \quad(\mathbf{e}(S)=\exp (\pi i \cdot \operatorname{trace}(S))),
$$

where $\mathbb{E}=\mathbb{E}_{n}$ is the set of all integral symmetric matrices of order $n$ with even entries on the principal diagonal (even matrices of order $n$ ), with constant Fourier coefficients $f(A)$ satisfying the relations

$$
f\left({ }^{\mathrm{t}} U A U\right)=(\operatorname{det} U)^{k} f(A) \quad\left(A \in \mathbb{E}, U \in \mathrm{GL}_{n}(\mathbb{Z})\right) .
$$

The Fourier series converges absolutely on the upper half-plane and converges uniformly when $Y=\Im Z \geq \varepsilon 1_{n}$ with $\varepsilon>0$.

2000 Mathematics Subject Classification: 11F46, 11F60, 11F66.

Key words and phrases: Hecke operators, Hecke-Shimura rings, Siegel modular forms, zeta functions of modular forms.

The author was supported in part by Russian Fund of Fundamental Researches, Grant 99-01-00099. 
For a given even positive definite matrix $Q$ of order $n$ and a Dirichlet character $\psi$, we associate to the form $F$ the Dirichlet series

$$
D_{F}(s, \psi, Q)=\sum_{M \in \Lambda \backslash \Delta} \frac{\psi(\operatorname{det} M) f\left(M Q^{\mathrm{t}} M\right)}{(\operatorname{det} M)^{s+k-1}},
$$

where

$$
\Lambda=\Lambda^{n}=\mathrm{SL}_{n}(\mathbb{Z}), \quad \Delta=\Delta^{n}=\left\{M \in \mathbb{Z}_{n}^{n} \mid \operatorname{det} M>0\right\} .
$$

The series converges absolutely and uniformly in a right half-plane of the complex variable $s$. We call the Dirichlet series (0.4) a theta-transform of the modular form $F$, since it can be obtained as the result of an integration of the product of $F$ with a suitable theta series of genus $n$ of the quadratic form with matrix $Q$. The corresponding integral representation, in particular, allows one to investigate analytical properties of the theta-transforms, which we do not touch in this work (see $[\mathrm{A}-\mathrm{K}(78)]$ and $[\operatorname{Shi}(94)]$ ).

On the other hand, it turns out that the theta-transforms of a common eigenfunction of a certain family of Hecke operators can be expressed in terms of the Euler product constructed by means of the corresponding eigenvalues, the so-called even zeta function of $F$, and Dirichlet $L$-functions. The most general results in this direction were obtained by G. Shimura [Shi(94)], who considered automorphic forms defined on the symplectic groups over totally real algebraic number fields. Shimura's general results are formulated in terms of common eigenfunctions of Hecke operators, but the question of existence of such functions, for $n>1$ and $q>1$, was left open.

Returning to the groups $\Gamma_{0}^{n}(q)$, it is well known that the subspace $\mathfrak{N}=\mathfrak{N}_{k}^{n}(q)$ of cusp forms of $\mathfrak{M}$ has a basis consisting of common eigenfunctions for all regular Hecke operators, i.e. operators whose multipliers are coprime with $q$ (see, for example, $[\operatorname{An}(87)$, Theorem 4.1.8]). The questions of diagonalization of singular Hecke operators, i.e. operators with multipliers dividing some power of $q$, were considered in [A-L(70)] for $n=1$, and in $[\operatorname{An}(99)]$ for $n=2$. In particular, it was proved in the last paper that a number of singular Hecke operators together with regular operators can be simultaneously diagonalized on certain invariant subspaces, subspaces of "new forms", of the spaces of cusp forms $\mathfrak{N}_{k}^{2}(q)$. In the present work we prove that, for square-free levels $q$, the list of Hecke operators diagonalizable on new forms includes also all operators sufficient for Euler factorization of the corresponding theta-transforms. This is done in Section 3 (Theorem 3.1). In Section 2 we extend our earlier techniques of Euler factorization developed in $[\operatorname{An}(78)]$ and $[\operatorname{An}(87), \S 4.3]$ for prime numbers not dividing the level $q$ in order to include also prime divisors of the level. Our elementary method leads to more explicit and concrete formulas than the general Shimura ap- 
proach and can be useful for further applications. Section 1 contains general information on Hecke operators.

I am grateful to Professor Eberhard Freitag of Heidelberg University, where this work was done and reported, for his kind invitation to participate in the work of the Heidelberg-Mannheim research group "Arithmetik" and for useful discussions on automorphic forms and related topics.

Notation. We fix the letters $\mathbb{N}, \mathbb{Z}, \mathbb{Q}$, and $\mathbb{C}$, as usual, for the set of positive rational integers, the ring of rational integers, the field of rational numbers, and the field of complex numbers, respectively.

If $\mathbb{A}$ is a set, $\mathbb{A}_{n}^{m}$ denotes the set of all $m \times n$ matrices with elements in $\mathbb{A}$. If $\mathbb{A}$ is a ring with the identity element, $1_{n}$ and $0_{n}$ denote the identity and zero elements of the ring $\mathbb{A}_{n}^{n}$. The transpose of a matrix $M$ is denoted by ${ }^{\mathrm{t}} M$. For two matrices $S$ and $N$ of appropriate sizes we set

$$
S[N]={ }^{\mathrm{t}} N S N .
$$

The numbers $k$ and $q$ for weight and level are arbitrary positive integers fixed throughout the work; the genus $n$ is an arbitrary fixed positive integer, except in Theorem 3.1 .

1. Hecke-Shimura rings and Hecke operators. For more details see $[\operatorname{An}(87)$, Chapters 3, 4] or $[\operatorname{An}(99)$, Chapter 1]. Let $S$ be a multiplicative semigroup and $G$ a subgroup of $S$ such that each double coset $G \sigma G$ with $\sigma \in S$ is a finite union of left $\operatorname{cosets} G \tau$ of $S$ modulo $G$. We then denote by $\mathcal{H}(G ; S)$ the Hecke-Shimura ring of the pair $G, S$ over $\mathbb{Q}$. We consider elements of the ring either as finite formal $\mathbb{Q}$-linear combinations of symbols $(\sigma)=(\sigma)_{G}$ bijectively corresponding to double cosets $G \sigma G \subset S$, or after replacing each $(\sigma)$ by the formal sum

$$
(\sigma)=\sum_{\tau \in G \backslash G \sigma G}(G \tau)
$$

of symbols $(G \tau)$ bijectively corresponding to different left cosets $G \tau$ contained in $G \sigma G$, as the corresponding formal linear combinations of the symbols associated to the left cosets. We shall call the symbols $(\sigma)=(\sigma)_{G}$ and $(G \tau)$ double classes and left classes (of $S$ modulo $G$ ), respectively.

In order to construct the required Hecke-Shimura rings we take the group $K$ defined by (0.1) and its triangular subgroup

$$
\Gamma_{0}=\Gamma_{0}^{n}=\left\{\left(\begin{array}{cc}
A & B \\
0_{n} & D
\end{array}\right) \in \operatorname{Sp}_{n}(\mathbb{Z})\right\}
$$

Then for the corresponding semigroups one can take the semigroup

$$
\Sigma=\Sigma^{n}=\left\{M \in \mathbb{Z}_{2 n}^{2 n} \mid{ }^{\mathrm{t}} M\left(\begin{array}{cc}
0_{n} & 1_{n} \\
-1_{n} & 0_{n}
\end{array}\right) M=\mu(M)\left(\begin{array}{cc}
0_{n} & 1_{n} \\
-1_{n} & 0_{n}
\end{array}\right), \mu(M)>0\right\}
$$


of integral symplectic matrices of order $n$ with positive multipliers $\mu(M)$ and its triangular subsemigroup

$$
\Sigma_{0}=\Sigma_{0}^{n}=\left\{\left(\begin{array}{cc}
A & B \\
0_{n} & D
\end{array}\right) \in \Sigma^{n}\right\} .
$$

The pairs $K, \Sigma$ and $K_{0}, \Sigma_{0}$ satisfy the necessary conditions and one can define the corresponding Hecke-Shimura rings

$$
\mathcal{H}=\mathcal{H}^{n}(q)=\mathcal{H}(K, \Sigma) \text { and } \quad \mathcal{H}_{0}=\mathcal{H}_{0}^{n}=\mathcal{H}\left(K_{0}, \Sigma_{0}\right) .
$$

The whole rings $\mathcal{H}$ and $\mathcal{H}_{0}$ are mainly used as playgrounds, where their various subrings can meet together. We recall that a double class $(M)$ of $\mathcal{H}$ is called regular (resp., singular) if the multiplier $\mu(M)$ is coprime with $q$ (resp., divides a power of $q$ ); the class is called reducible if each left coset $K M$ contained in the double coset $K M K$ has a representative in the semigroup $\Sigma_{0}$. The set of all finite linear combinations of regular (resp., singular, reducible) double classes obviously form a subring of $\mathcal{H}$, called, respectively, the regular, singular, and reducible subring (see $[\operatorname{An}(99), \S 1.6]$ ).

It is easy to see that, for each element $T=\sum_{i} c_{i}\left(K M_{i}\right)$ of the reducible subring $\mathcal{H}_{\text {red }}$ of $\mathcal{H}$ with all $M_{i}$ in $\Sigma_{0}$, the linear combination $\mathbf{i}(T)=$ $\sum_{i} c_{i}\left(\Gamma_{0} M_{i}\right)$ is independent of the choice of $M_{i} \in K M_{i} \cap \Sigma_{0}$, belongs to $\mathcal{H}_{0}$, and the map

$$
\mathbf{i}: \mathcal{H}_{\text {red }} \rightarrow \mathcal{H}_{0}
$$

is a homomorphic embedding, the parabolic embedding of the ring $\mathcal{H}_{\text {red }}$.

The Hecke-Shimura rings naturally act on the corresponding linear spaces by Hecke operators. First, for matrices $M=\left(\begin{array}{ll}A & B \\ C & D\end{array}\right)$ of $\Sigma$, we define the Petersson operators of weight $k$ on complex-valued functions on the upper half-plane $\mathbb{H}$ by

$$
\begin{aligned}
\| M & =\left\|M_{k}: F \rightarrow F\right\| M \\
& =\mu(M)^{n k-n(n+1) / 2} \operatorname{det}(C Z+D)^{-k} F\left((A Z+B)(C Z+D)^{-1}\right) .
\end{aligned}
$$

The Petersson operators satisfy

$$
\left\|\left(M M^{\prime}\right)=\right\| M \| M^{\prime} \quad \text { and } \quad F \| M=F \quad \text { if } F \in \mathfrak{M} \text { and } M \in K .
$$

As a representation space for the ring $\mathcal{H}$, we shall take the space of modular forms $\mathfrak{M}=\mathfrak{M}_{k}^{n}(q)$, and for the ring $\mathcal{H}_{0}$ the space $\mathfrak{F}=\mathfrak{F}_{k}^{n}$ of all absolutely convergent Fourier series on $\mathbb{H}=\mathbb{H}_{n}$ of the form (0.2) with constant Fourier coefficients satisfying (0.3). For $F$ in $\mathfrak{M}$ (resp., $\mathfrak{F})$ and $T=\sum_{i} c_{i}\left(K M_{i}\right) \in \mathcal{H}$ (resp., $\left.T=\sum_{i} c_{i}\left(K_{0} M_{i}\right) \in \mathcal{H}_{0}\right)$ we set

$$
F\|T=F\|_{k} T=\sum_{i} c_{i} F \| M_{i}
$$

with the Petersson operators $\| M_{i}$. It follows from (1.2) that the operators (1.3), called Hecke operators, are independent of the choice of the rep- 
resentatives $M_{i} \in K M_{i}$, map the corresponding spaces into themselves, and satisfy $\left\|T T^{\prime}=\right\| T \| T^{\prime}$. It is clear that $\mathfrak{M} \subset \mathfrak{F}$, and this embedding is compatible with the embedding (1.1) of the Hecke-Shimura rings in the sense that

$$
F\|T=F\| \mathbf{i}(T) \quad\left(\forall F \in \mathfrak{M} \text { and } T \in \mathcal{H}_{\text {red }}\right) .
$$

Note also that the subspace $\mathfrak{N}$ of cusp forms of $\mathfrak{M}$ is invariant under all the Hecke operators of $\mathcal{H}$.

If $F$ in $\mathfrak{M}$ (resp., $\mathfrak{F}$ ) is a function with Fourier coefficients $f(A)$ $\left(A \in \mathbb{E}_{n}, A \geq 0\right.$ ), and $T \in \mathcal{H}$ (resp., $\left.T \in \mathcal{H}_{0}\right)$, we denote by $(f \| T)(A)$ the Fourier coefficients of the function $F \| T$. The map

$$
T: f \mapsto f \| T
$$

is clearly a linear representation of the corresponding ring, the representation on Fourier coefficients.

In the forthcoming computations, it will be convenient to use certain extensions of the rings $\mathcal{H}$ and $\mathcal{H}_{0}$ rather than the rings themselves. We recall that if $\mathbb{A}$ is an associative ring with unity and $S$ is a multiplicative subset of the center of $\mathbb{A}$ containing the unity and not containing zero divisors, then by using the standart procedure of localization with respect to the multiplicative system $S$, one can construct the least extension $\mathbb{A}\left[S^{-1}\right]$ of $\mathbb{A}$ where all elements of $S$ are invertible.

The following scalar double classes of $\mathcal{H}$ (resp., $\mathcal{H}_{0}$ ):

$$
[a]=\left(a 1_{2 n}\right)_{K} \quad\left(\operatorname{resp} .,\left(a 1_{2 n}\right)_{\Gamma_{0}}\right) \quad(a \in \mathbb{N})
$$

clearly satisfy the relations

$$
[a b]=[a][b], \quad[a](M)=(a M) \quad\left(\forall a \in \mathbb{N}, M \in \Sigma\left(\text { resp., } \Sigma_{0}\right)\right),
$$

and therefore form a multiplicative system with the required properties. We denote by

$$
\mathcal{H}^{\prime}=\mathcal{H}\left[[a]^{-1} \mid a \in \mathbb{N}\right], \quad \mathcal{H}_{0}^{\prime}=\mathcal{H}_{0}\left[[a]^{-1} \mid a \in \mathbb{N}\right]
$$

the corresponding localizations. Similarly, one can consider localizations of subrings of $\mathcal{H}$ and $\mathcal{H}_{0}$ with respect to multiplicative subsystems of scalar classes contained in the subrings. We shall refer to the localizations as the quotient rings, or just quotients. Note that the scalar classes have the following simple action on Fourier coefficients of functions in $\mathfrak{M}$ and $\mathfrak{F}$ :

$$
(f \|[a])(A)=a^{n(k-n-1)} f(A) \quad(a \in \mathbb{N}, A \in \mathbb{E}, A \geq 0) .
$$

2. Euler factorization of theta-transforms. In this section we deduce Euler factorizations of the theta-transforms (0.4) of a modular form $F$ of the space $\mathfrak{M}=\mathfrak{M}_{k}^{n}(q)$, assuming that $F$ is an eigenfunction for an explicit family of Hecke operators. 
Lemma 2.1. Let $\mathcal{C}_{+}\left(\right.$resp., $\left.\mathcal{C}_{-}\right)$be the set of all linear combinations, contained in $\mathcal{H}_{0}$, of double classes modulo $\Gamma_{0}$ of matrices of the form

$$
M=U(\mu, D)=\left(\begin{array}{cc}
\mu^{\mathrm{t}} D^{-1} & 0_{n} \\
0_{n} & D
\end{array}\right) \in \Sigma_{0}
$$

satisfying the conditions $\operatorname{det} D>0$ and

$$
\mu \mid d(D)^{2} \quad\left(\text { resp., } l(D)^{2} \mid \mu\right),
$$

where $d(D)$ is the greatest common divisor of the elements of $D$, and $l(D)$ is the least positive integer such that the matrix $l(D) D^{-1}$ is integral. Then $\mathcal{C}_{+}$ (resp., $\mathcal{C}_{-}$) is a commutative subring of $\mathcal{H}_{0}$. The double classes of the matrices of the form (2.1) satisfying (2.2) act on Fourier coefficients $f$ of functions in $\mathfrak{F}$ by the formulas

$$
(f \|(M))(A)=\mu^{n k-n(n+1)}(\operatorname{det} D)^{n+1-k} \sum_{D^{\prime} \in \Lambda \backslash \Lambda D \Lambda} f\left(\mu^{-1} A\left[{ }^{\mathrm{t}} D^{\prime}\right]\right)
$$

(resp.,

$$
\left.(f \|(M))(A)=\mu^{n k-n(n+1) / 2}(\operatorname{det} D)^{-k} \sum_{\substack{D^{\prime} \in \Lambda \backslash \Lambda D \Lambda \\ A\left[{ }^{\mathrm{t}} D^{\prime}\right] \in \mu \mathbb{E}}} f\left(\mu^{-1} A\left[{ }^{\mathrm{t}} D^{\prime}\right]\right)\right),
$$

where $\Lambda=\mathrm{SL}_{n}(\mathbb{Z})$.

Proof. See $[\operatorname{An}(87)$, Proposition 3.4.4, Theorem 3.4.7, and Lemma 4.2.7].

Lemma 2.2. For $a=1,2, \ldots$, set

$$
t_{+}(a)=t_{+}^{n}(a)=\sum_{\substack{D \in \Lambda \backslash \Delta / \Lambda \\ \operatorname{det} D=a}}[a]^{-1}\left(U\left(a^{2}, a D\right)\right) \in \mathcal{H}_{0}^{\prime},
$$

where $\Lambda$ and $\Delta$ are given by (0.5). Then:

(1) The elements (2.4) belong to the quotient $\mathcal{C}_{+}^{\prime}$ of the ring $\mathcal{C}_{+}$relative to the multiplicative system (1.5); in particular, the elements commute with each other.

(2) If $a$ and $b$ are coprime, then

$$
t_{+}(a b)=t_{+}(a) t_{+}(b) .
$$

(3) For the Fourier coefficients $f(A)$ of any function in $\mathfrak{F}$, and $a \in \mathbb{N}$, one can write

$$
\left(f \| t_{+}(a)\right)(A)=a^{n+1-k} \sum_{M \in \Lambda \backslash \Delta, \operatorname{det} M=a} f\left(A\left[{ }^{\mathrm{t}} M\right]\right) \quad(A \in \mathbb{E}, A \geq 0) .
$$

Proof. The lemma follows easily from [An(87), Lemma 4.3.20]. 
LEMMA 2.3. For each prime number $p$, the following identity holds in the ring of formal power series over $\mathcal{C}_{+}^{\prime}$ :

$$
\sum_{\delta=0}^{\infty} p^{-\delta n} t_{+}\left(p^{\delta}\right) v^{\delta}=\left(\sum_{i=0}^{n}(-1)^{i} x_{i}(p) v^{i}\right)^{-1},
$$

where

$$
x_{i}(p)=x_{i}^{n}(p)=p^{i(i-1) / 2-i n}[p]_{\Gamma_{0}}^{-1}\left(U\left(p^{2}, p D_{i}(p)\right)\right)_{\Gamma_{0}}
$$

with

$$
D_{i}(p)=D_{i}^{n}(p)=\left(\begin{array}{cc}
1_{n-i} & 0_{n} \\
0_{n} & p 1_{i}
\end{array}\right) .
$$

Proof. The identity (2.5) is (3.5.74) of $[\mathrm{An}(87)$, Lemma 3.5.24]. The formulas (2.6) follow from (3.5.34) of [An(87), Proposition 3.5.17].

Lemma 2.4. Let $\mathcal{C}_{+}(q)$ be the set of all finite linear combinations of the double classes modulo $\Gamma_{0}$ of elements of the form (2.1) satisfying the condition $\mu \mid d(D)^{2}$ and such that $\operatorname{det} D$ divides some powers of $q$. Then $\mathcal{C}_{+}(q)$ is a subring of $\mathcal{C}_{+}$, and the mapping

$$
\mathcal{C}_{+}(q) \ni T=\sum_{i} a_{i}\left(\Gamma_{0} M_{i}\right) \mapsto \widetilde{T}=\sum_{i} a_{i}\left(K M_{i}\right)
$$

defines an isomorphism of the ring $\mathcal{C}_{+}(q)$ onto the subring $\widetilde{\mathcal{C}}_{+}(q)$ of the reducible subring $\mathcal{H}_{\mathrm{red}}$ of $\mathcal{H}$ consisting of all finite combinations of double classes modulo $K$ of the same elements. The assertion remains true also for the quotients $\mathcal{C}_{+}^{\prime}(q)$ and $\widetilde{\mathcal{C}_{+}^{\prime}}(q)$ of the rings relative to the scalar classes corresponding to positive integers dividing some powers of $q$.

Proof. The lemma follows from [An(99), Theorem 3.3].

Next we recall the definitions of local subrings of $\mathcal{H}^{\prime}$ and of Rankin polynomials over the local subrings. For more details see, for example, $[\operatorname{An}(87)$, $\S 3.3-\S 3.5]$. The (maximal) regular reducible subring of $\mathcal{H}$ can be described as the Hecke-Shimura ring of the pair $K, R^{*}(K)$, where

$R^{*}(K)=\left\{M=\left(\begin{array}{cc}A & B \\ C & D\end{array}\right) \in \Sigma \mid \mu(M)\right.$ is coprime with $\left.q, C \equiv 0(\bmod q)\right\}$ ([An(99), Lemma 2.1]). For a prime number $p$ not dividing the level $q$, we set

$$
R_{p}(K)=\left\{M \in R^{*}(K) \mid \mu(M) \text { is a power of } p\right\},
$$

and define the local (reducible) $p$-subring $\mathcal{H}_{p}^{\prime}$ of $\mathcal{H}^{\prime}$ as the quotient relative to the system of scalar classes, for $a=1, p, p^{2}, \ldots$, of the ring $\mathcal{H}\left(K, R_{p}(K)\right)$. We now define the spherical map

$$
\Omega: \mathcal{H}_{p}^{\prime} \mapsto \mathbb{Q}\left[x_{0}^{ \pm 1}, x_{1}^{ \pm 1}, \ldots, x_{n}^{ \pm 1}\right],
$$


where $x_{0}, x_{1}, \ldots, x_{n}$ are (commuting) independent variables over $\mathbb{Q}$. If

$$
T=\sum_{i} c_{i}[p]^{-a_{i}}\left(K M_{i}\right)
$$

is an element of $\mathcal{H}_{p}^{\prime}$, one can assume that each $M_{i}$ has the form

$$
M_{i}=\left(\begin{array}{cc}
\mu_{i}{ }^{\mathrm{t}} D_{i}^{-1} & B_{i} \\
0_{n} & D_{i}
\end{array}\right)
$$

where $D_{i}$ is an upper triangular matrix with powers of $p$, say, $p^{d_{i 1}}, \ldots, p^{d_{i n}}$, on the principal diagonal and $\mu_{i}=p^{\delta_{i}}$. Then we set

$$
\Omega(T)=\sum_{i} c_{i} x_{0}^{-2 a_{i}+\delta_{i}} \prod_{j=1}^{n}\left(x_{j} p^{-j}\right)^{d_{i j}-a_{i}} .
$$

The spherical map is a homomorphic embedding and one can show that the image $\Omega\left(\mathcal{H}_{p}^{\prime}\right)$ contains, in particular, all the coefficients of the polynomial in $v$

$$
\prod_{j=1}^{n}\left(1-x_{j} v\right)\left(1-x_{j}^{-1} v\right)=\sum_{i=0}^{2 n}(-1)^{i} r_{i}\left(x_{1}, \ldots, x_{n}\right) v^{n} .
$$

The polynomial over $\mathcal{H}_{p}^{\prime}$ given by

$$
R_{p}(v)=R_{p}^{n}(v)=\sum_{i=0}^{2 n}(-1)^{i} r_{i}(p) v^{i} \quad \text { with } \quad \Omega\left(r_{i}(p)\right)=r_{i}\left(x_{1}, \ldots, x_{n}\right)
$$

is called the Rankin p-polynomial.

The coefficients of the Rankin polynomials satisfy the relations

$$
r_{2 n-i}(p)=r_{i}(p) \quad(i=0, \ldots, 2 n) ; \quad r_{0}(p)=r_{2 n}(p)=\mathbf{1},
$$

where $\mathbf{1}=\left(1_{2 n}\right)_{K}$ is the unit element of $\mathcal{H}$. The coefficients

$$
r_{1}(p)=r_{1}^{n}(p), \quad \ldots, \quad r_{n}(p)=r_{n}^{n}(p)
$$

together with the scalar classes $[p]=\left(p 1_{2 n}\right)_{K}$ and $[p]^{-1}$ generate the even subring of $\mathcal{H}_{p}^{\prime}$.

If $F \in \mathfrak{M}$ is an eigenfunction for the Hecke operators

$$
\left\|r_{1}(p)=\right\|_{k} r_{1}^{n}(p), \quad \ldots, \quad\left\|r_{n}(p)=\right\|_{k} r_{n}^{n}(p)
$$

with the eigenvalues $\lambda\left(r_{i}(p)\right)$, then the polynomial

$$
\lambda\left(R_{p}\right)(v)=\sum_{i=0}^{2 n}(-1)^{i} \lambda\left(r_{i}(p)\right) v^{i} \quad\left(\lambda\left(r_{2 n-i}(p)\right)=\lambda\left(r_{i}(p)\right)\right)
$$

has the factorization of the form

$$
\lambda\left(R_{p}\right)(v)=\prod_{j=1}^{n}\left(1-\alpha_{j}(p) v\right)\left(1-\alpha_{j}(p)^{-1} v\right) .
$$


The numbers $\alpha_{1}(p)^{ \pm 1}, \ldots, \alpha_{n}(p)^{ \pm 1}$ are called the Satake $p$-parameters of the eigenfunction $F$.

The main result of this section is the following theorem.

TheOREM 2.5. Let $F \in \mathfrak{M}=\mathfrak{M}_{k}^{n}(q)$ be a modular form with Fourier expansion (0.2). Suppose that $F$ is an eigenfunction for the Hecke operators $\|T=\|_{k} T$ with eigenvalues

$$
\lambda(T)=\lambda_{F}(T)
$$

for all elements $T$ of the form

$$
\widetilde{x}_{i}(p)=\widetilde{x_{i}^{n}}(p)=p^{i(i-1) / 2-i n}[p]_{K}^{-1}\left(U\left(p^{2}, p D_{i}(p)\right)\right)_{K} \quad(i=1, \ldots, n),
$$

where $U(\mu, D)$ and $D_{i}(p)=D_{i}^{n}(p)$ are the matrices defined in (2.1) and (2.6), for all prime divisors of the level $q$ (singular prime numbers), and for all elements $T$ of the form (2.8) for all prime numbers not dividing the level (regular prime numbers). Then, for each Dirichlet character $\psi$ and each even positive definite matrix $Q$ of order $n$, the following formal factorization of the theta-transform (0.4) holds:

$$
D_{F}(s, \psi, Q)=Z^{+}(s, \psi, F)\left(\prod_{p \nmid q} B_{p}\left(\psi(p) p^{-s}, Q\right)\right) X_{F}(s, \psi, Q) .
$$

Here and below p runs over all prime numbers satisfying the corresponding conditions, and the following notation is used:

$$
Z^{+}(s, \psi, F)=Z_{s}^{+}(s, \psi, F) Z_{r}^{+}(s, \psi, F)
$$

with

$$
Z_{s}^{+}(s, \psi, F)=\prod_{p \mid q}\left(\sum_{i=0}^{n}(-1)^{i} \lambda_{F}\left(\widetilde{x}_{i}(p)\right)\left(\psi(p) p^{-s}\right)^{i}\right)^{-1}
$$

and

$$
Z_{r}^{+}(s, \psi, F)=\prod_{p \nmid q}\left(\prod_{j=1}^{n}\left(1-\alpha_{j}(p, F) \psi(p) p^{-s}\right)\left(1-\alpha_{j}(p, F)^{-1} \psi(p) p^{-s}\right)\right)^{-1},
$$

where $\alpha_{j}(p, F)^{ \pm 1}$ are the Satake p-parameters of $F$, is the even zeta function of the eigenfunction $F$;

$$
B_{p}(v, Q)=B_{p}^{n}(v, Q)
$$

are polynomials in $v$ depending only on the $\mathrm{GL}_{n}\left(\mathbb{F}_{p}\right)$-equivalence class of the quadratic form with matrix $Q$ considered over the finite field $\mathbb{F}_{p}$ of $p$ elements and satisfying the following rules: $B_{p}^{n}(v, Q)=B_{p}^{m}\left(v, Q^{\prime}\right)$ if the quadratic form with matrix $Q$ is equivalent modulo $p$ to a quadratic form in $m<n$ variables with matrix $Q^{\prime}$, but if the form is nondegenerate modulo $p$, 
then

$$
B_{p}^{n}(v, Q)= \begin{cases}(1+v)\left(1-\chi_{Q}(p) p^{-l} v\right) \prod_{i=1}^{l-1}\left(1-p^{-2 i} v^{2}\right) & \text { if } n=2 l \\ (1+v) \prod_{i=1}^{l}\left(1-p^{-2 i} v^{2}\right) & \text { if } n=2 l+1\end{cases}
$$

where, for even $n, \chi_{Q}$ denotes the Dirichlet character of the quadratic form with matrix $Q$; finally,

$$
X_{F}(s, \psi, Q)=\left(f \| \prod_{\substack{p \nmid q \\ p \mid \operatorname{det} Q}}\left(\sum_{i=0}^{n}(-1)^{i} x_{-i}(p) \psi\left(p^{i}\right) p^{-i s}\right)\right)(Q)
$$

with the elements

$$
x_{-i}(p)=x_{-i}^{n}(p)=p^{i(i-1) / 2-i n}[p]_{\Gamma_{0}}^{-1}\left(U\left(p^{2}, D_{n-i}(p)\right)\right)_{\Gamma_{0}}
$$

of the quotient ring $\mathcal{C}_{-}^{\prime}$ of the ring $\mathcal{C}_{-}$defined in Lemma 2.1, whose action on the Fourier coefficients can be described by the formulas

$$
\left(f \| x_{-i}(p)\right)(Q)=p^{i(k-n)+\left(i^{2}-i\right) / 2} \sum_{\substack{D \in \Lambda \backslash \Lambda D_{n-i} \Lambda \\ A\left[{ }^{\mathrm{t} D}\right] \in p^{2} \mathbb{E}_{n}}} f\left(p^{-2} A\left[{ }^{\mathrm{t}} D\right]\right),
$$

in particular,

$$
\left(f \| x_{-i}(p)\right)(Q)=0 \quad \text { if } p^{2 i} \nmid \operatorname{det} Q .
$$

The Dirichlet series on the left side of (2.10) and the infinite products on the right side converge absolutely and uniformly in any half-plane of the form $\Re s>(2 \tau-1) k+n+1+\varepsilon$ with $\varepsilon>0$, where $\tau=1 / 2$ if $F$ is a cusp form, and $\tau=1$ otherwise.

Proof. By considering the Fourier coefficients $f(A)$ of the function $F$ as values of a function $f$ belonging to the space of Fourier coefficients of functions in $\mathfrak{M} \subset \mathfrak{F}$ and using the action (1.4) of the Hecke-Shimura rings on the spaces of Fourier coefficients and Lemma 2.2, one can write

$$
\begin{aligned}
D_{F}(s, \psi, Q) & =\sum_{a=1}^{\infty} \frac{\psi(a)\left(f \| a^{-n} t_{+}(a)\right)(Q)}{a^{s}} \\
& =\left(f \| \prod_{p \text { prime }} \sum_{\delta=0}^{\infty} p^{-\delta n} t_{+}\left(p^{\delta}\right) \psi(p)^{\delta} p^{-\delta s}\right)(Q),
\end{aligned}
$$

which, by Lemma 2.3, can be written as

$$
D_{F}(s, \psi, Q)=\left(f \| \prod_{p \text { prime }}\left(\sum_{i=0}^{n}(-1)^{i} x_{i}(p)\left(\psi(p) p^{-s}\right)^{i}\right)^{-1}\right)(Q) .
$$

Note that the order of $p$-factors of the last product is unessential, by Lemma 2.2(1). Consider first the action of singular $p$-factors. Since the mapping (2.7) is clearly compatible with the action of the corresponding Hecke 
operators on the functions in $\mathfrak{M} \subset \mathfrak{F}$ and their Fourier coefficients, and the function $f$ together with $F$ is an eigenfunction for the Hecke operators corresponding to the elements (2.9), we have, for each singular prime, the identity

$$
\begin{aligned}
f \|\left(\sum_{i=0}^{n}(-1)^{i} x_{i}(p)\left(\psi(p) p^{-s}\right)^{i}\right)^{-1} & \\
& =f \|\left(\sum_{i=0}^{n}(-1)^{i} \widetilde{x}_{i}(p)\left(\psi(p) p^{-s}\right)^{i}\right)^{-1} \\
& =\left(\sum_{i=0}^{n}(-1)^{i} \lambda_{F}\left(\widetilde{x}_{i}(p)\right)\left(\psi(p) p^{-s}\right)^{i}\right)^{-1} f .
\end{aligned}
$$

Let us now turn to the regular primes. If $p$ is one, then by the hypothesis of the theorem,

$$
\begin{aligned}
& \left(\sum_{i=0}^{2 n}(-1)^{i} \lambda_{F}\left(r_{i}(p)\right)\left(\psi(p) p^{-s}\right)^{i}\right) f \|\left(\sum_{i=0}^{n}(-1)^{i} x_{i}(p)\left(\psi(p) p^{-s}\right)^{i}\right)^{-1} \\
& \quad=f \|\left(\sum_{i=0}^{2 n}(-1)^{i} r_{i}(p)\left(\psi(p) p^{-s}\right)^{i}\right)\left(\sum_{i=0}^{n}(-1)^{i} x_{i}(p)\left(\psi(p) p^{-s}\right)^{i}\right)^{-1},
\end{aligned}
$$

where $r_{i}(p)$ are the coefficients of the Rankin polynomial $R_{p}$. According to [An(87), Theorem 3.5.23],

$$
R_{p}(v)\left(\sum_{i=0}^{n}(-1)^{i} x_{i}(p) v^{i}\right)^{-1}=\left(\sum_{i=0}^{n}(-1)^{i} x_{-i}(p) v^{i}\right) B_{p}(v),
$$

where $x_{i}(p)$ are the elements (2.6) and

$$
B_{p}(v)=B_{p}^{n}(v)=\sum_{i=0}^{n}(-1)^{i} b_{i}(p) v^{i}
$$

is a polynomial over the ring $\mathcal{H}_{0}^{\prime}$. Then, by [An(87), Proposition 4.2.24], for every function $g$ in the space of Fourier coefficients of functions in $\mathfrak{F}$ and every even matrix $A$ of order $n$,

$$
\left(g \| B_{p}(v)\right)(A)=\sum_{i=0}^{n}(-1)^{i}\left(g \| b_{i}(p)\right)(A) v^{i}=B_{p}(v, A) g(A),
$$

where $B_{p}(v, A)=B_{p}^{n}(v, A)$ are polynomials in $v$ independent of $g$. From (2.13)-(2.15) we obtain the identity

$$
\begin{aligned}
& \quad\left(f \|\left(\sum_{i=0}^{n}(-1)^{i} x_{i}(p)\left(\psi(p) p^{-s}\right)^{i}\right)^{-1}\right)(Q) \\
& =\lambda_{F}\left(R_{p}\right)\left(\psi(p) p^{-s}\right)^{-1} B_{p}\left(\psi(p) p^{-s}, Q\right) \sum_{i=0}^{n}(-1)^{i}\left(f \| x_{-i}(p)\right)(Q)\left(\psi(p) p^{-s}\right)^{i}
\end{aligned}
$$


valid for each regular prime $p$, where the polynomial

$$
\lambda_{F}\left(R_{p}\right)(v)=\sum_{i=0}^{2 n}(-1)^{i} \lambda_{F}\left(r_{i}(p)\right) v^{i}
$$

can be expressed through the Satake $p$-parameters $\alpha_{i}(p, F)^{ \pm 1}$ by

$$
\lambda_{F}\left(R_{p}\right)(v)=\prod_{j=1}^{n}\left(1-\alpha_{j}(p, F) v\right)\left(1-\alpha_{j}(p, F)^{-1} v\right) .
$$

From (2.11), by using first the identities (2.12) for each singular prime and then (2.16) for each regular prime, we finally get the formal identity (2.10). Explicit formulas for the polynomials $B_{p}^{n}(v, Q)$ were obtained in $\left[\operatorname{An}(87)\right.$, Theorem 4.2.27]. The formulas for the action of the elements $x_{-i}(p)$ on the Fourier coefficients follow from (2.3). The convergence of the series and products follows from the known estimates of Fourier coefficients of modular forms. For more details on regular primes and the convergence, see [An(87), Proof of Theorem 4.3.19].

REMARKS. (1) The formal identity of the theorem remains valid with the same proof for an arbitrary completely multiplicative function $\psi: \mathbb{N} \rightarrow \mathbb{C}$, not necessarily a Dirichlet character.

(2) Similar results can be obtained for modular forms with Dirichlet characters modulo $q$ for the groups $\Gamma_{0}^{n}(q)$. For regular primes this was done in $[\mathrm{An}(87)$, Theorem 4.3.19]. The singular primes can be treated analogously to the above, with the groups $\Gamma_{0}^{n}(q)$ replaced by the subgroups

$$
\left\{M=\left(\begin{array}{cc}
A & B \\
C & D
\end{array}\right) \in \Gamma_{0}^{n}(q) \mid \operatorname{det} D \equiv 1(\bmod q)\right\} .
$$

(3) The product $\prod_{p \text { prime }} B_{p}\left(\psi(p) p^{-s}, Q\right)^{-1}$ looks very much like a "zeta function" with the character $\psi$ of the quadratic form with matrix $Q$.

\section{Diagonalization of centralizers of singular Frobenius ele-} ments. According to $[\operatorname{An}(87)$, Theorem 4.1.8], the subspace of cusp forms of $\mathfrak{M}_{k}^{n}(q)$ has a basis consisting, in particular, of common eigenfunctions for all Hecke operators corresponding to the coefficients (2.8) of the Rankin polynomials $R_{p}(v)$, for all regular prime numbers $p$. Therefore, in order to apply the above theorem, one has first to consider the question of diagonalization of Hecke operators corresponding to (2.9) for singular primes. It follows from Lemma 2.4 and [An(87), Propositions 3.4.4, 3.4.7, and Theorem 3.2.17] that the elements (2.9) together with the scalar classes $[p]^{ \pm 1}$ for all $p \mid q$ generate the ring $\widetilde{\mathcal{C}_{+}^{\prime}}(q)$ defined in Lemma 2.4. This ring, by [An(99), Theorem 3.3], 
can be characterized as the centralizer of the singular Frobenius elements

$$
\Pi(a)=\Pi_{K}^{n}(a)=\left(\left(\begin{array}{cc}
1_{n} & 0_{n} \\
0_{n} & a 1_{n}
\end{array}\right)\right)_{K} \quad \text { with } \quad a \mid q^{\infty} \quad\left(K=\Gamma_{0}^{n}(q)\right)
$$

$\left(a \mid q^{\infty}\right.$ means that $a$ divides a power of $\left.q\right)$ in the singular reducible subring of $\mathcal{H}^{\prime}=\mathcal{H}^{n}(q)$. In [An(99), Chapter 4] a subspace $\mathbf{N}=\mathbf{N}_{k}^{n}(q)$ of "new" cusp forms of $\mathfrak{N}_{k}^{n}(q)$ was introduced for arbitrary $n$ (for $n=1$ it was done in $[\mathrm{A}-\mathrm{L}(70)])$. In particular, it was proved there that, for $n=2$, the space of new forms has a basis of common eigenfunctions for all regular Hecke operators and a number of singular Hecke operators including all the singular Frobenius elements. Here we shall prove that in this case the list of Hecke operators diagonalizable on new forms also includes the operators corresponding to all elements of local centralizers of Frobenius elements $\Pi(p)$ for all primes $p$ dividing $q$ but not $q / p$ (unramified primes). But first we recall related definitions and results from [An(99)] (that paper was originally issued in English as a preprint of Max-Planck-Institut für Mathematik, Preprint series 1998 (118)). For brevity, below we refer to, e.g., [An(99), Theorem 1.9] or $[\operatorname{An}(99)$, formula (3.66)] just as [Theorem 1.9] or [(3.66)].

For arbitrary $n$, we consider the quotient $\mathcal{H}^{\prime}$ defined by (1.12) and its action (1.3) on the space $\mathfrak{N}$ of cusp forms. The star map of the ring $\mathcal{H}^{\prime}$ is a linear map defined on the double classes by

$$
(M)^{*}=\left(\mu(M) M^{-1}\right), \quad\left([a]^{-1}\right)^{*}=[a]^{-1} \quad(M \in \Sigma, a \in \mathbb{N}) .
$$

The star map is an antiautomorphism of the second order of $\mathcal{H}^{\prime}$ ([Theorem 1.9(1)]). The space $\mathfrak{N}$ is a finite-dimensional Hilbert space with respect to the Petersson scalar product $\mathfrak{N} \ni F, F^{\prime} \mapsto\left(F, F^{\prime}\right)$ (see, for example, [§1.4]). The Hecke operators $\| T$ and $\| T^{*}$ are conjugate with respect to this scalar product:

$$
\left(F \| T, F^{\prime}\right)=\left(F, F^{\prime} \| T^{*}\right) \quad\left(F, F^{\prime} \in \mathfrak{N}, T \in \mathcal{H}^{\prime}\right)
$$

([Theorem 1.9(2)]). It turns out that, for each prime divisor $p$ of $q$ not dividing $q / p$ (singular unramified prime), the Frobenius element $\Pi(p)$ is invertible in the singular subring $\mathcal{H}_{\mathrm{s}}^{\prime}$ of $\mathcal{H}^{\prime}$. This allows one to define an embedding of the (regular reducible) local $p$-subring $\mathcal{H}_{p}^{\prime}(q / p)$ of the HeckeShimura ring $\mathcal{H}^{\prime}(q / p)$ for the group $\Gamma_{0}^{n}(q / p)$ into the ring $\mathcal{H}_{\mathrm{s}}^{\prime}$ (see [§3.3]). We denote by

$$
\widetilde{\mathcal{H}_{p}^{\prime}}(q / p) \subset \mathcal{H}_{\mathrm{s}}^{\prime}=\mathcal{H}_{\mathrm{s}}^{\prime}(q)
$$

the image of this embedding.

For each singular prime $p$, the subspace of p-new forms of $\mathfrak{N}=\mathfrak{N}_{k}^{n}(q)$ can be defined by

$$
\mathbf{N}_{p}=\mathbf{N}_{p}(\mathfrak{N})=\left\{F \in \mathfrak{N} \mid F \| \Pi(p)^{i} \tau(p)=0 \text { for } i=0,1, \ldots, 2^{n}-1\right\},
$$


if $p^{2} \nmid q$, and by

$$
\mathbf{N}_{p}=\mathbf{N}_{p}(\mathfrak{N})=\{F \in \mathfrak{N} \mid F\|\tau(p)=0, F\| \Pi(p) \tau(p)=0\}
$$

if $p^{2} \mid q$, where

$$
\tau(p)=\tau^{n}(p, q)=\left[K^{\prime}: K\right]^{-1} \sum_{M_{i} \in K \backslash K^{\prime}}\left(K M_{i}\right)
$$

with $K^{\prime}=\Gamma_{0}^{n}(q / p)$ and $K=\Gamma_{0}^{n}(q)$ is the trace-idempotent of the pair $K \subset K^{\prime}$. The subspace of new forms of $\mathfrak{N}$ is then defined by

$$
\mathbf{N}=\mathbf{N}(\mathfrak{N})=\bigcap_{p \mid q} \mathbf{N}_{p}
$$

(see $[\S 4.2])$.

Now we have the following theorem on diagonalization of Hecke operators on new forms.

Theorem 3.1. (1) The subspace $\mathbf{N}=\mathbf{N}\left(\mathfrak{N}_{k}^{n}(q)\right)$ of new forms of $\mathfrak{N}_{k}^{n}(q)$ is invariant under all the Hecke operators corresponding to elements of local regular reducible $p$-subrings $\mathcal{H}_{p}^{\prime}$ of $\mathcal{H}^{\prime}$ for all regular prime numbers $p$.

(2) For every $n$ and each prime number $p$ satisfying $p^{2} \mid q$, the Hecke operators $\| \Pi(p)$ and $\| \Pi^{*}(p)$ on $\mathbf{N}$ are equal to the zero operator.

(3) If $n=2$, then, for all singular unramified prime numbers $p$, the space $\mathbf{N}$ is invariant under all the Hecke operators corresponding to the following elements of the singular Hecke-Shimura ring: elements of the local singular unramified p-rings $\widetilde{\mathcal{H}_{p}^{\prime}}(q / p)$; singular Frobenius elements $\Pi(p)$ and their dual elements $\Pi^{*}(p)$; all elements of the form (2.9).

(4) If $n=2$, then there is a basis of $\mathbf{N}$ consisting of common eigenfunctions for all the Hecke operators listed in (1)-(3). In particular, if $q$ is square-free, then $\mathbf{N}$ has a basis of common eigenfunctions for all the Hecke operators listed in Theorem 2.5 above.

Proof. Part (1) is proved in [Theorem 4.6]. Part (2) for $\| \Pi(p)$ is proved in [Lemma 4.13]. By [Proposition 3.12(2), (4)], we have $\Pi^{*}(p)=(\Pi(p) \tau(p))^{*}=$ $\tau(p) \Pi^{*}(p)$, which implies the assertion for $\| \Pi^{*}(p)$.

In order to prove (3) and (4), we note that in this case the set (2.9), for each singular prime $p$, reduces to the two elements $\widetilde{x_{1}^{2}}(p)$ and $\widetilde{x_{2}^{2}}(p)$ which, according to (1.7), can be replaced by

$$
\Pi_{1}\left(p^{2}\right)=U\left(p^{2}, p D_{1}^{2}(p)\right)_{K} \quad \text { and } \quad U\left(p^{2}, p D_{2}^{2}(p)\right)_{K}=\Pi\left(p^{2}\right) .
$$

By using [Theorem 3.3(1)], one can show that $\Pi\left(p^{2}\right)=\Pi(p)^{2}$. Then, by taking into account [Theorem 4.15], it is sufficient to prove that the element $\Pi_{1}\left(p^{2}\right)$, for each singular unramified prime $p$, can be expressed as a polynomial in elements of the ring $\widetilde{\mathcal{H}_{p}^{\prime}}(q / p)$ and the Frobenius element $\Pi(p)$. By comparing the coefficients of $v$ in both parts of the factorization $[(3.66)]$, we 
get the relation

$$
\widetilde{T}(p)=p^{3}[p] \Pi(p)^{-1}+p \Pi(p)^{-1} \Pi_{1}\left(p^{2}\right)+\Pi(p),
$$

where $\widetilde{T}(p)$ is the image in the ring $\widetilde{\mathcal{H}_{p}^{\prime}}(q / p)$ of the double class

$$
T(p)_{K^{\prime}}=\left(\left(\begin{array}{cc}
1_{2} & 0_{2} \\
0_{2} & p 1_{2}
\end{array}\right)\right)_{K^{\prime}} \in \mathcal{H}_{p}^{\prime}(q / p)
$$

with $K^{\prime}=\Gamma_{0}^{2}(q / p)$. We conclude that

$$
\Pi_{1}\left(p^{2}\right)=\frac{1}{p}\left(\Pi(p) \widetilde{T}(p)-\Pi(p)^{2}-p^{3}[p]\right) .
$$

The theorem follows.

REMARK. It is easy to see that the last of the elements (2.9) has the form

$$
\widetilde{x_{n}^{n}}(p)=p^{-n(n+1) / 2}[p]^{-1} \Pi(p)^{2} .
$$

Then, according to (2), the corresponding Hecke operator on the space of new forms of $\mathfrak{N}_{k}^{n}(q)$ is the zero operator, for all primes $p$ with $p^{2} \mid q$. So, for such primes, the denominators of the corresponding $p$-factors of the even zeta function have degree in $\psi(p) p^{-s}$ less than $n$. In particular, for $n=2$, the denominators have the form $1-\lambda_{F}\left(\widetilde{x}_{1}(p)\right) \psi(p) p^{-s}$.

\section{References}

$[\operatorname{An}(78)] \quad$ A. N. Andrianov, Euler expansions of theta-transforms of Siegel modular forms of degree n, Mat. Sb. 105 (1978), 291-341 (in Russian); English transl.: Math. USSR-Sb. 34 (1978), 259-300.

[An(87)] -, Quadratic Forms and Hecke Operators, Springer, Berlin, 1987.

[An(99)] - Singular Hecke-Shimura rings and Hecke operators on Siegel modular forms, Algebra i Analiz 11 (1999), 1-68 (in Russian); English transl.: St. Petersburg Math. J. 11 (2000), 931-987.

[A-K(78)] A. N. Andrianov and V. L. Kalinin, On the analytic properties of standard zeta functions of Siegel modular forms, Mat. Sb. 106 (1978), 323-339; English transl.: Math. USSR-Sb. 35 (1979), 1-17.

[A-L(70)] A. O. L. Atkin and J. Lehner, Hecke operators on $\Gamma_{0}(m)$, Math. Ann. 185 (1970), 134-160.

[Shi(94)] G. Shimura, Euler products and Fourier coefficients of automorphic forms on symplectic groups, Invent. Math. 116 (1994), 531-576.

Steklov Mathematical Institute

St. Petersburg Branch

Fontanka 27

191011 St. Petersburg, Russia

E-mail: anandr@pdmi.ras.ru 